\title{
All-on- 4 dental implant concept in immediate rehabilitation of failing dentition-a prospective study to evaluate the efficacy and cost effectiveness in Indian population
}

\begin{abstract}
Background: The close proximity of anatomical structures in atrophied ridges makes placement of conventional implants difficult. However the use of tilted implants and immediate restoration has made rehabilitation of these ridges simpler: by eliminating various ridge augmentation procedures and the waiting period prior to prosthetic rehabilitation.
\end{abstract}

Purpose: To evaluate the stability of implants used to restore the edentulous ridges by clinical and radiological assessment, change in the marginal bone level and patient satisfaction.

Materials \& methods: This study included 15 edentulous jaws. Sixty implants were placed using the All-on-Four concept. Immediate loading of implants was done with a full arch acrylic prosthesis (intermediate prosthesis).

Results: This study concluded that out of 60 implants that were placed during the course of the treatment 56 implants were stable for the time the patients were followed up. Four tilted implant failures were seen.

Conclusion: Implant survival rate and the prosthetic survival rate was $93.3 \%$. A mean marginal bone loss of $0.49 \mathrm{~mm}$ and $0.09 \mathrm{~mm}$ was seen in the mandible and maxilla. The marginal bone loss was higher around the tilted implants $(0.37 \mathrm{~mm})$.

Keywords: all-on-four, edentulous jaws, failing dentition, tilted implants, initial torque, immediate function, immediate loading, acrylic prosthesis, complete arch, patient satisfaction
Volume II Issue I - 2020

\section{Sthita Gurrala,Vikas Dhupar, Francis Akkara \\ Department of Oral \& Maxillofacial Surgery, Goa Dental College \& Hospital, India}

Correspondence: Sthita Gurrala, Senior Resident, Department of Oral \& Maxillofacial Surgery Goa Dental College \& Hospital, Bambolim, Goa, Pincode 403202, India, Tel +919552399760, Email gurralasthit@gmail.com

Received: January 21, 2020 | Published: January 29, 2020

\section{Introduction}

Effective rehabilitation of failing dentition with dental implants is a multi-step process which involves a period of edentulism before the patient can be rehabilitated prosthetically. This period may be objectionable by many who are undergoing a transition from natural dentition to implant supported prosthesis. The all-on-4 concept developed by Paulo Malo has been in clinical use since 1998. This concept effectively uses 4 implants placed in strategic position to support prosthesis with minimal cost implication and at the same time serving to immediately rehabilitate the patient with an interim prosthesis. This avoids the period of edentulism and social embarrassment for the patients with failing dentition.

This concept involves full arch rehabilitation using only four implants- two straight implants placed anteriorly and two implants placed posterior supporting provisional, fixed and immediately loaded full-arch prosthesis. Tilted implants eliminate the need for grafting and also increase the bone-implant contact. ${ }^{1,2}$ Malo et al. ${ }^{2}$ reported a cumulative implant survival rate of $100 \%$ with immediate extraction, placement and loading of implants after 1 year. ${ }^{3}$ Full mouth dental rehabilitation became a reality in atrophic and failing dentition patients. The objective of this prospective study was to evaluate the efficacy of All-on-Four treatment concept in Indian population of patients with edentulous jaws and failing dentition. The study was designed to evaluate factors such as primary stability of the implants, patient satisfaction and affordability and also clinical and radiological assessment of changes in the bone level.

\section{Methods and materials}

This study was approved by the institutional ethical committee board and the design was in adherence to the Helsinki declaration for ethical human trial. The individuals participating in this clinical investigation were all part of a prospective clinical trial aiming to evaluate all-on-four implant related treatment outcome. A total of 15 edentulous jaws which met the inclusion criteria were included in this study. All these patients reported to the Goa Dental College \& Hospital for rehabilitation of residual resorbed ridges or failing dentition between December 2015 and September 2017 were included in the study. Patients included in the study had an age range of 30 75 years. All the patients included were partially edentulous with remaining dentition failing or with severely resorbed ridges which required complete prosthetic rehabilitation. We excluded from the study any patient who had radiation or bisphosphonate therapy in the past. Total of eleven patients ( 5 males and 6 females) were treated after they fulfilled the inclusion criterion. We used Nobel Active Implants (Nobel Biocare) in all patients. The surgical placements 
of implants were performed by a single surgeon to eliminate any operator bias. Impressions of the edentulous ridge or the dentulous ridge were taken and study models were fabricated. This was followed by a radiographic evaluation which included a CBCT (Figure 1-5). With the available data, treatment plan was discussed with the patients and other treatment options were also given to the patients. The dental casts were used to fabricate a temporary or immediate prosthesis for the patient. For patients in whom immediate extractions were planned, the temporary dentures were fabricated on the dental cast models by removing the teeth from the cast. Surgical planning was done using the Noble Clinician software. A surgical guide was fabricated utilizing the data and the implants were strategically placed according to it A total of 60 implants were placed out of which 24 were placed in the maxilla and 36 in the mandible. All surgical procedures were performed under local anesthesia after administration of prophylactic antibiotics. The surgical procedure for placement of implants was in accordance with the standard procedure as described by Paulo Malo.

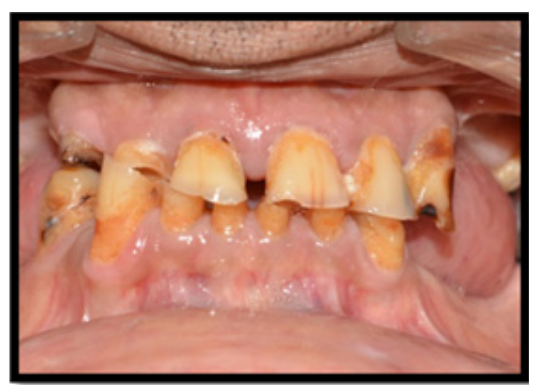

Figure I Clinical photograph.

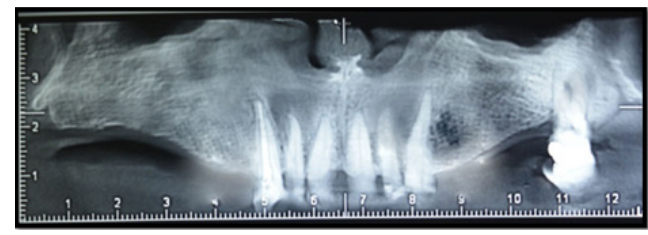

Figure 2 CBCT image.

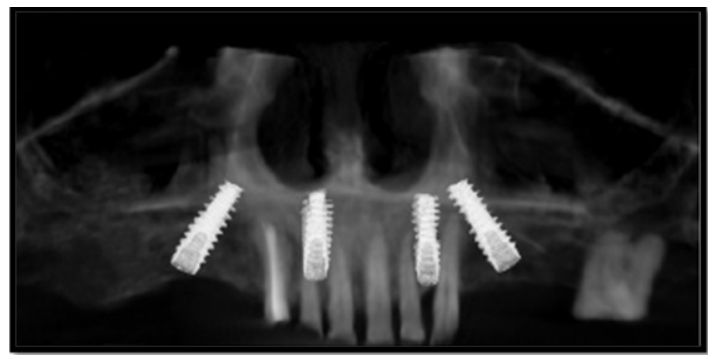

Figure 3 Virtual planning of placement of implants.

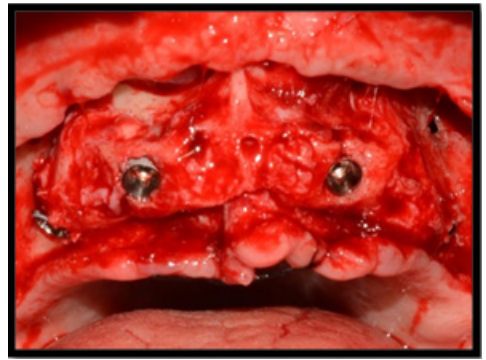

Figure 4 Implants in situ.

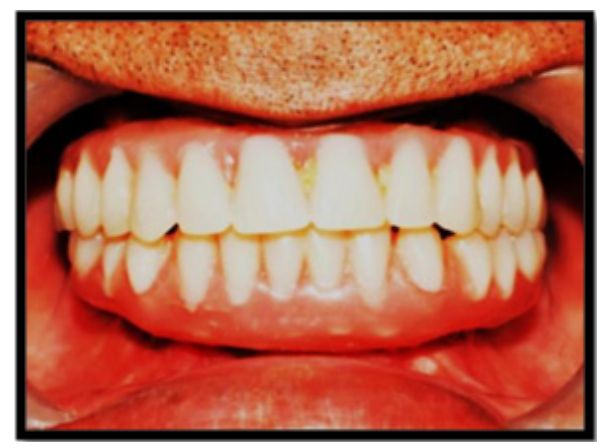

Figure $\mathbf{5}$ Intermediate prosthesis.

\section{Prosthetic procedure}

To index the position of the multiunit abutments Aquasil light body addition silicone was used to line the undersurface of the denture. Access openings were made in the denture for the temporary coping cylinders which were the attached to the multiunit abutments. The denture was passively seated onto the temporary coping cylinders. Temporary coping cylinders were removed and trimmed to the appropriate height and reattached. Rubber dam was placed around the cylinders to prevent the acrylic material from entering the sutured sites. The access holes of the temporary coping cylinders were blocked with Aquasil putty addition silicone material to prevent acrylic from entering the hole. The seating and proper alignment of the denture was verified using the pre-operative bite registration. Pickup of the temporary coping cylinders was done by adding cold cure acrylic around the cylinders. Once the acrylic was set, the denture along with the temporary copings was unscrewed with a Unigrip driver. Healing caps were attached to the multiunit abutment while the provisional prosthesis was being finished. The palate, borders, and phalanges of the prosthesis were trimmed. A distal cantilever of $3 \mathrm{~mm}$ was maintained. An ovate pontic contour was created for ease of maintenance/ hygiene in the maxillary and mandibular dentures. The sharp edges were smoothened. The denture was polished and delivered on the same day of the surgery. The screws were torqued to $15 \mathrm{Ncm}$ using a torque wrench. The access holes were blocked with Teflon tape and an implant protected occlusion was obtained.

All patients were included in a prospective follow-up protocol and were evaluated at intervals of 48 hours, 7 days, 3 months and 6 months postoperatively (Figure 6). Radiographic evaluation was done immediately following surgery, at 3 months and at 6 months of follow up. The prostheses were removed at each follow-up appointment to perform the clinical assessments.

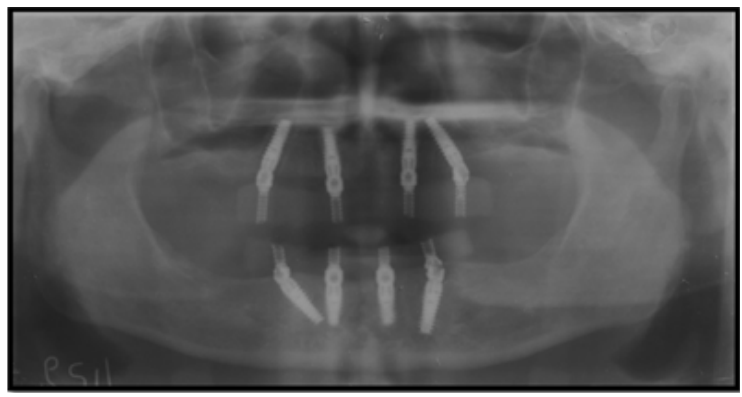

Figure 6 Post-operative radiograph. 
The clinical data recorded for evaluation of primary stability of implants were in terms of initial torque, percussion, mobility and probing depth. The marginal bone loss was assessed by comparing radiographs taken immediately, in 3 months and 6 months. The reference point for the reading was the implant platform (the horizontal interface between the implant and the multiunit abutment). Height of the marginal bone was measured on the mesial and distal surface of the implant.

\section{Results}

A total of 60 implants (Nobel Active Ti unite) were placed in 11 patients 5 males and 6 females, mean age at surgery 59.4 years, range $30-75$ years]. Six maxillary and nine mandibular prostheses were delivered. All prostheses were supported by four implants. All implants could be seated with an insertion torque of at least $35 \mathrm{Ncm}$. All provisional prostheses could be delivered the same day of surgery as planned.

Implant distribution according to implant length is detailed in Tables 1-3. The outcome analysis is based on those 11 patients that have had their prosthesis in function for at least 6 months at the time of the present clinical report. Nine prostheses (36 implants) were immediately loaded in the mandible. The follow-up range was 3-6 months. Patients had different types of opposing dentition: removable prostheses, natural teeth and fixed prostheses on natural teeth (1 case), fixed prostheses on natural teeth (one case), and implant-supported bridges ( 1 case), and implant-supported prostheses (four cases).

Table I Implant distribution according to implant length

\begin{tabular}{lllll}
\hline \multicolumn{5}{l}{ Implant distribution according to implant length } \\
\hline \multicolumn{5}{l}{ Length of the implants } \\
\hline Maxilla & 10 & 11.5 & 13 & 15 \\
Number of implants & 3 & 7 & 9 & 5 \\
Mandible & 11.5 & 13 & 15 & \\
Number of implants & 14 & 8 & 14 \\
\hline
\end{tabular}

Table 2 Marginal bone loss assessment

\begin{tabular}{lllllll}
\hline Site & & Mean & N & Std. deviation & Std. error mean \\
\hline \multirow{2}{*}{ Mandible } & Pair I & Marginal bone level immediate post-operative & 1.49 & 18 & 0.71 & 0.17 \\
& & Marginal bone level 6 months post-operative & 1.03 & 18 & 0.6 & 0.14 \\
\multirow{2}{*}{ Maxilla } & \multirow{2}{*}{ Pair I } & Marginal bone level immediate post-operative & 0.81 & 12 & 0.69 & 0.19 \\
& & Marginal bone level post-operative & 0.72 & 12 & 0.34 & 0.09 \\
\hline
\end{tabular}

Table 3 Denture satisfaction questionnaire

\begin{tabular}{lll}
\hline Question I & Frequency & Percent \\
\hline Satisfied & 14 & 93.3 \\
Neither Satisfied Nor Dissatisfied & 1 & 6.7 \\
Total & 15 & 100 \\
Question 2 & & \\
Better with Implant supported Denture & 12 & 80 \\
No Improvement & 3 & 20 \\
Total & 15 & 100 \\
Question 3 & & \\
Better with Implant supported Denture & 12 & 80 \\
No Improvement & 3 & 20 \\
Total & 15 & 100 \\
Question 4 & & \\
Better with Implant supported Denture & 8 & 53.3 \\
No Improvement & 7 & 46.7 \\
Total & 15 & 100 \\
Question 5 & 15 & \\
Satisfied & & 100 \\
\hline
\end{tabular}


Six prostheses (24 implants) were placed in the maxilla out of which five were immediately loaded. The opposing dentitions were removable prostheses, implant-supported prostheses (four cases). No complications occurred during the surgical phase. Three tilted implants in the maxilla (in one male and two female patients) and one tilted implant in the mandible (in one male patient) failed within 6 months of function, due to mobility. In all cases implant failure did not compromise prosthesis function. All implants could be successfully replaced. No late failures have been recorded to date. No relation was found between implant failure and the opposing dentition. The overall cumulative implant survival after 6 months was 93.3\%.The only prosthetic complication in this study was the fracture of the acrylic prosthesis that occurred in one case in the mandible. The average marginal bone loss after 6 months from implant placement was 0.09 in the maxilla (24 implants in 6 patients) and $0.46 \mathrm{~mm}$ in the mandible (36 implants in 9 patients). The marginal bone loss was statistically significant in the mandible and statistically insignificant in the maxilla.

\section{Discussion}

This prospective study aimed at evaluating the clinical and radiographic outcomes of a technique for the immediate rehabilitation of patients with failing dentition. The preliminary results indicate that such technique may lead to good patient satisfaction, excellent clinical results and affordability. In this study the distribution of the maxilla: mandible was $2: 3$. Out of 36 implants placed in the mandible one failed with an overall survival rate of $97.2 \%$. Success rate in the mandible is in concurrence with most of the studies. This is because there is good quality of bone present in the anterior region of the mandible. Similar findings were seen with mandibular implant survival rate of $99 \%{ }^{4}$ Friberg et al. ${ }^{5}$ stated that there was also a predominance of mandibles. One implant that failed can be attributed to the fact that the patient had a failing dentition and was also a controlled diabetic. ${ }^{6,7}$ However out of 24 implants placed in the maxilla three failed resulting in an overall success rate of $87.5 \%$. Out of the three implant failures in the maxilla, two failures occurred in a patient who had bruxism whereas one failure was seen in a patient with no specific cause. In the maxilla the placement of implants is highly technique sensitive. This is due to the presence of the maxillary sinus and the nasal cavity. Bone is more cancellous in the maxilla, therefore it is mandatory to achieve bicortical anchorage during implant placement. $^{8}$

Among the 4 implant failures, all the implants were tilted implants. A meta-analysis by Chrcanovic et al. ${ }^{9}$ showed that the position of the implants whether axial or tilted did not affect the implant survival rate. ${ }^{9}$ Bellini et al. ${ }^{10}$ stated that no significant difference in stress was found in the axial and tilted implants. However increased stress on the titled implants was attributed to an increase in the cantilever length. ${ }^{10}$ In a single cohort study done by Agnini A et al, implant survival rate for axial implants was $97.56 \%$ and for tilted implants was $100 \% .^{5}$ The high success rates of tilted implants have made it a viable option. ${ }^{11-13}$ However implant failure of tilted implants were higher in this study stating the contrary.

Out of the 60 implants placed in this study 56 were loaded immediately whereas 4 implants were loaded after a week (early loading). The survival rate of the implants with early loading was $100 \%$. The finding of this study is in accordance to Kronstrom M et al. ${ }^{14}$ who noted that early loading of implants had a high success rate. Implants survival was assessed based on clinical and radiographic findings. At the time of surgery implants were evaluated by the initial torque achieved. This was followed by evaluation of mobility, percussion and probing depth at 3 months and 6 months of follow up. Mobility was present in 4 implants. All 4 implants had severe horizontal mobility greater than $0.5 \mathrm{~mm}$. Probing depth was assessed around all the implants. More than $5 \mathrm{~mm}$ of probing depth was seen in the implants that failed to get osseointegrated.

The radiographic assessment was carried out by comparing the marginal bone level on an orthopentamogram taken immediately after surgery and at 6 months of follow up. The mean marginal bone loss was $0.46 \mathrm{~mm}$ in the mandible and 0.09 in the maxilla seen below the implant abutment interface. The marginal bone loss is higher in this study for tilted implants $0.37 \mathrm{~mm} \pm 0.20$ as compared to the axial implants $0.27 \mathrm{~mm} \pm 0.85$, the marginal bone loss around tilted implants is higher and is statistically significant $(\mathrm{p}=0.539>0.05)$.

Temporary denture fabricated prior to the surgical procedure was used to load the implants. The dentures were modified to limit the distal cantilever length to $5 \mathrm{~mm}$. Implant protected occlusion was achieved in the patients. No intra-operative surgical complication was reported in any patient. Common complications described in literature are fracture of the prosthesis and screw loosening. In this study acrylic prosthesis fractured in one patient which was retrieved and repaired without any risk to the implants. Prosthetic survival rate in this study is $93.3 \%{ }^{15,16}$ Loosening of the screw was a complication commonly seen in this study which may be a result of not achieving adequate torque while tightening the screws.

Overall patients were satisfied with this treatment protocol. The patients preferred implant supported prostheses over removable dentures. This finding is in accordance to the patient satisfaction rate seen in patients rehabilitated with the All-on-four treatment concept. We conclude by stating all-on-four concept is a viable alternative to conventional implants in patients having edentulous jaws or failing dentition with compromised bone having high esthetic and functional needs.

\section{Funding details}

None.

\section{Acknowledgments}

None.

\section{Conflicts of interest}

The authors declare that there is no conflict of interest.

\section{References}

1. Maló P, Rangert B, Nobre M. All-on-Four” immediate-function concept with Brånemark System implants for completely edentulous mandibles: A retrospective clinical study. Clin Implant Dent Relat Res. 2003;5 Suppl 1:2-9.

2. Maló P, Rangert B, Nobre M. All-on-4 immediate-function concept with Brånemark System implants for completely edentulous maxillae: A 1-year retrospective clinical study. Clin Implant Dent Relat Res. 2005;7 Suppl 1:S88-S94.

3. Malo P, de Araujo Nobre M, Rangert B. Implants placed in immediate function in periodontally compromised sites: a five-year retrospective and one-year prospective study. J Prosthet Dent. 2007;97(Suppl 6):S8695.

4. Friberg B, Jemt T, Lekholm U. Early failures in 4,641 consecutively placed Branemark dental implants: A study from stage 1 surgery to the connection of completed prostheses. Int J Oral Maxillofac Implants. 1991;6(2):142-146. 
5. Agnini A, Agnini AM, Romeo D, et al. Clinical investigation on axial versus tilted implants for immediate fixed rehabilitation of edentulous arches: Preliminary results of a single cohort study. Clin Implant Dent Relat Res. 2014;16(4):527-539.

6. Malo P, de Araújo Nobre M, Lopes A, et al. A longitudinal study of the survival of All-on-4 implants in the mandible with up to 10 years of follow-up. J Am Dent Assoc. 2011;142(3):310-320.

7. Attard NJ, Zarb GA. Immediate and early implant loading protocols: A literature review of clinical studies. J Prosthet Dent. 2005;94(3):242-58.

8. Hasegawa T. Survival of Branemark System Mk III implants and analysis of risk factors associated with implant failure. Int $J$ Oral Maxillofac Surg. 2017;46(2):267-273.

9. Chrcanovic BR, Albrektsson T, Wennerberg A. Tilted versus axially placed dental implants: A meta-analysis. J Dent. 2015;43(2):149-170.

10. Bellini CM. Comparison of tilted versus nontilted implantsupported prosthetic designs for the restoration of the edentuous mandible: A biomechanical study. Int $J$ Oral Maxillofac Implants. 2009;24(3):511-517.
11. Del Fabbro M, Bellini CM, Romeo D, et al. Tilted implants for the rehabilitation of edentulous jaws: A systematic review. Clin Implant Dent Relat Res. 2012;14(4):612-621.

12. Cavalli N, Barbaro B, Spasari D, et al. Tilted implants for full-arch rehabilitations in completely edentulous maxilla: A retrospective study. Int J Dent. 2012;2012:180379.

13. Ata-Ali J, Penarrocha-Oltra D, Candel-Marti E, et al. Oral rehabilitation with tilted dental implants: A meta-analysis. Med Oral Patol Oral Cir Bucal. 2012;17:e582-e587.

14. Kronström M, Widbom $\mathrm{T}$, Löfquist $\mathrm{LE}$, et al. Early functional loading of conical Branemark implants in the edentulous mandible: A 12-monthfollow-up clinical report. J Prosthet Dent. 2003;89(4):335-340.

15. Randow K, Ericsson I, Nilner K, et al. Immediate functional loading of Branemark dental implants. An 18-month clinical follow-up study. Clin Oral Implants Res. 1999;10(1):8-15.

16. Ericsson I, Randow K, Nilner K, et al. Early functional loading of Branemark dental implants: 5-year clinical follow-up study. Clin Implant Dent Relat Res. 2000;2(2):70-77. 